\title{
Improved Heuristic Drift Elimination with Magnetically-aided Dominant Directions (MiHDE) for Pedestrian Navigation in Complex Buildings
}

\author{
A.R. Jiménez, F. Seco, F. Zampella, J.C. Prieto and J. Guevara
}

\begin{abstract}
The main problem of Pedestrian Dead-Reckoning (PDR) using only a body-attached IMU is the accumulation of heading errors. The heading provided by magnetometers in indoor buildings is in general not reliable and therefore it is commonly not used. Recently, a new method was proposed called Heuristic Drift Elimination (HDE) that minimizes the heading error when navigating in buildings. It assumes that the majority of buildings have their corridors parallel to each other, or they intersect at right angles, and consequently most of the time the person walks along a straight path with a heading constrained to one of four possible directions. In this paper we study the performance of HDE-based methods in complex buildings, i.e. with pathways also oriented at $45^{\circ}$, long curved corridors, and wide areas where non-oriented motion is possible. We explain how the performance of the original HDE method can be deteriorated in complex buildings, and also, how severe errors can appear in case of false matches with the building's dominant directions. Although magnetic compassing indoors has a chaotic behavior, in this paper we analyze large data-sets in order to study the potential use that magnetic compassing has to estimate the absolute yaw angle of a walking person. Apart from these analysis, this paper also proposes an improved HDE method called MiHDE (Magnetically-aided Improved Heuristic Drift Elimination), that is implemented over a PDR framework that uses foot-mounted inertial navigation with an Extended Kalman Filter (EKF). The EKF is fed with the MiHDE-estimated orientation error, gyro bias corrections, as well as the confidence over that corrections. We experimentally evaluated the performance of the proposed MiHDE-based PDR method, comparing it with the original HDE implementation. Results show that both methods perform very well in ideal orthogonal narrow-corridor buildings, and MiHDE outperforms HDE for non-ideal trajectories (e.g. curved paths) and also makes it robust against potential false dominant direction matchings.
\end{abstract}

\section{INTRODUCTION}

The main problem of Pedestrian Dead-Reckoning (PDR) using only a body-attached IMU (Inertial Measurement Unit) is the accumulation of heading errors. The heading provided by magnetometers in indoor buildings is in general not reliable, and consequently is not commonly used in the PDR community for accurate navigation. Recently, a new method was proposed by Borenstein and Ojeda [1] called Heuristic Drift Elimination (HDE) that minimizes the heading error when navigating in buildings. It assumes that the majority of buildings have dominant directions defined by the orientation of their corridors; consequently a person walks most of the time along straight-line paths parallel to these dominant directions. Abdulrahim et al. [2] exploit the same building's dominant directions assumption, but they implement the HDE idea in a totally different way. See Fig. 1 for a simplified description of a PDR algorithm, which is similar to the Abdulrahim et al. implementation, that uses the Dominant Direction-based heuristic.

The implementation in [1] uses a feedback control loop at the output of a vertically-aligned gyroscope. In the loop there is an integration stage to obtain the heading angle from the gyroscopic angular rate, and then this angle is compared to one of the main building orientations. The heading error is fed into a binary integral (I)-controller, whose output is an estimation of the slow-changing bias of the gyroscope, which is subtracted from the measured gyroscopic angular rate to obtain an "unbiased" version of the gyro's angular rate. The I-controller has a gain proportional to the size of the step, so the gyro bias is computed preferably with long steps.

The implementation in [2] uses an inertial navigation or INS-based framework to directly integrate triads of accelerometer and gyroscopic signals. This INS mechanization is corrected by a complementary Kalman filter (see [3] and [4] for INSbased PDR implementation details). The heading difference between the dominant directions of the building and that of the user's stride (heading error) is fed as a measurement into the Kalman filter. When the Stride Length (SL) is shorter than $0.3 \mathrm{~m}$, the heading correction is deactivated.

These two works ([1], [2]) exploit the concept of the dominant directions in a building but do not use magnetometers to give information about the absolute heading or yaw angle of the person while is walking. They state that this information is not reliable enough and avoid its use. However, recently some proposals try to obtain benefits from perturbed magnetic information using complex arrays of magnetometer to partially compensate yaw errors [5], and also, capturing the total magnetic field change at foot stances in order to improve the estimation of gyro biases [6]. Other approaches are possible, as will be proposed in this paper, where we use improved heuristics based on building's dominant directions, and also yaw information obtained by mid-term magnetic compassing. 


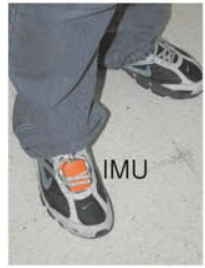

a)
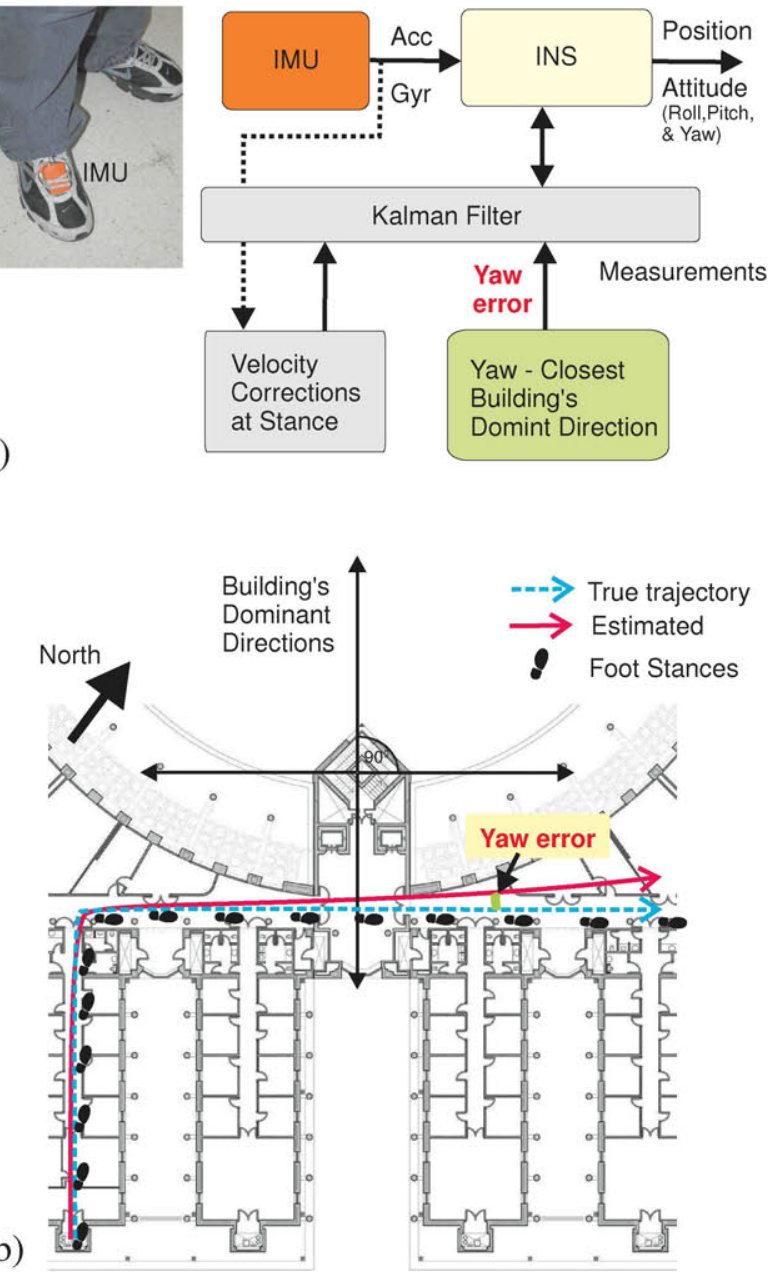

Fig. 1. Pedestrian Dead-Reckoning (PDR) with the HDE heuristic. a) The basic INS-based PDR approach extended with the HDE heuristic that uses information from the main Building's Dominant Directions (green color block). b) Trajectory in a building with 2 dominant directions (horizontal \& vertical). Note that there is an error in Yaw, specially at the end of the trajectory, between the estimated yaw and the yaw of the closest dominant direction (horizontal). This Yaw error can be used by the HDE heuristic to correct the INS estimation.

In this paper (section II) we analyze the benefits of the above-cited HDE implementations, but also their limitations, which include a damage in the navigation solution when used in complex buildings (e.g. the one in Fig. 2a), which has curved corridors, pathways oriented other than $90^{\circ}$, and wide areas for non-oriented motion. The section III analyzes the limits and potential benefits found in magnetic compassing; it is shown the chaotic behavior of short-term compassing but it is experimentally analyzed how this data can be used in a mid- or long-term to correct the absolute yaw angle. Based on the conclusions obtained in the last two sections, we present in section IV an improved HDE method, called MiHDE (Magnetically-aided Improved Heuristic Drift Elimination), that although similar somehow to the Abdulrahim et al. implementation [2] includes a motion analysis block to detect straight-line paths and an adaptive on-line confidence estimator for the heading corrections. This method also includes a procedure to improve the gyro bias updates, and also a method to select the correct dominant direction of the building that takes into account the the mid-term yaw errors obtained from the magnetometers. Finally, the section V presents some experimental results with synthetically generated and real paths that contains straight, curved and multiple-loop trajectories in the test building.

\section{HDE: BENEFITS AND LIMITATIONS}

\section{A. HDE Benefits}

HDE methods estimate the non-deterministic slow-variant bias of the gyro's angular rate. Therefore, they make the heading error to be observable. In fact the heading observability is almost as good as if a digital compass were used (assuming no magnetic disturbances). An HDE-based PDR solution basically eliminates the error in heading, and consequently, it reduces the positioning error. For example in [1] a $0.33 \%$ error of the Total Traveled Distance (TTD) is obtained, and in [2] the reported error is just $0.1 \%$ of the TTD.

Fig. 2b shows a PDR trajectory estimation example using HDE in an "ideal" floor that includes narrow long corridors at $0,45^{\circ}$ and $90^{\circ}$ orientations. If the least angular difference between the dominant directions in a building is denoted by $\Delta$, then this difference is $45^{\circ}$ for the building under test in this paper $\left(\Delta=45^{\circ}\right)$. In Fig. $2 \mathrm{~b}$ is also included the non-HDE aided solution (IEZ) that is dominated by the uncorrected gyro drift in heading. As can be seen, HDE is an extraordinary method to navigate indoors.

\section{B. HDE Limitations}

HDE uses a progressive correction of the gyro bias in order to obtain a robust operation even under temporal paths along non-ideal paths (curved or straight paths out of the dominant directions). If walking more than 30-60 seconds along non-ideal paths, then HDE can deteriorate the navigation solution as Borenstein states [1]. In Fig. 3 it is graphically shown the damaging actions of HDE for two non-ideal paths. The deformation of the true trajectory is progressive, not too severe, but causes a slight error in positioning and heading that can be accumulated.

The progressive error accumulation of HDE method over non-ideal trajectories, could in principle cause the estimated trajectory to match a wrong dominant direction. If this occurs then the estimation is severely deteriorated since the trajectory aligns with a wrong direction and positioning completely fails. Although the problem of wrong matching it is unlikely to occur especially if $\Delta \geq 45^{\circ}$ and the non-ideal paths are not too long, in principle under certain circumstances it could appear (e.g. very long non-ideal paths, usage of low performance IMU, poor initial bias estimations,...). We will propose methods to detect these situations, avoid wrong matchings to a dominant direction, and to alleviate its estimation consequences. 


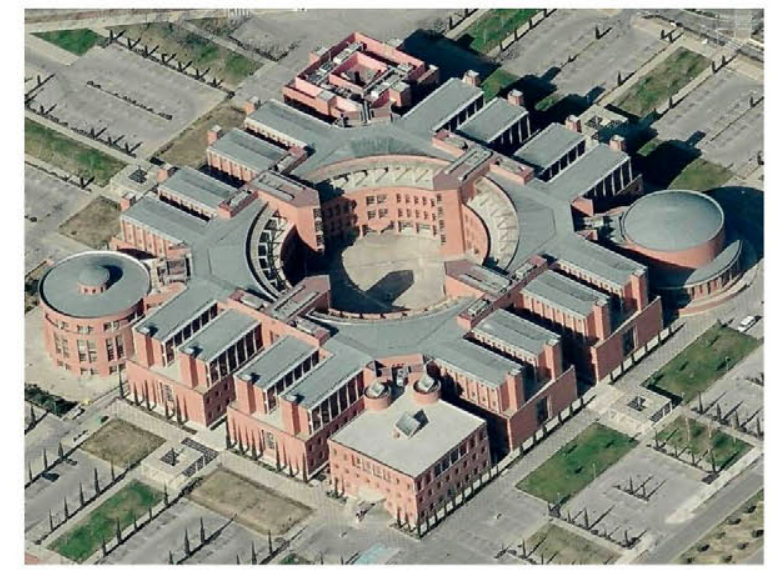

a)

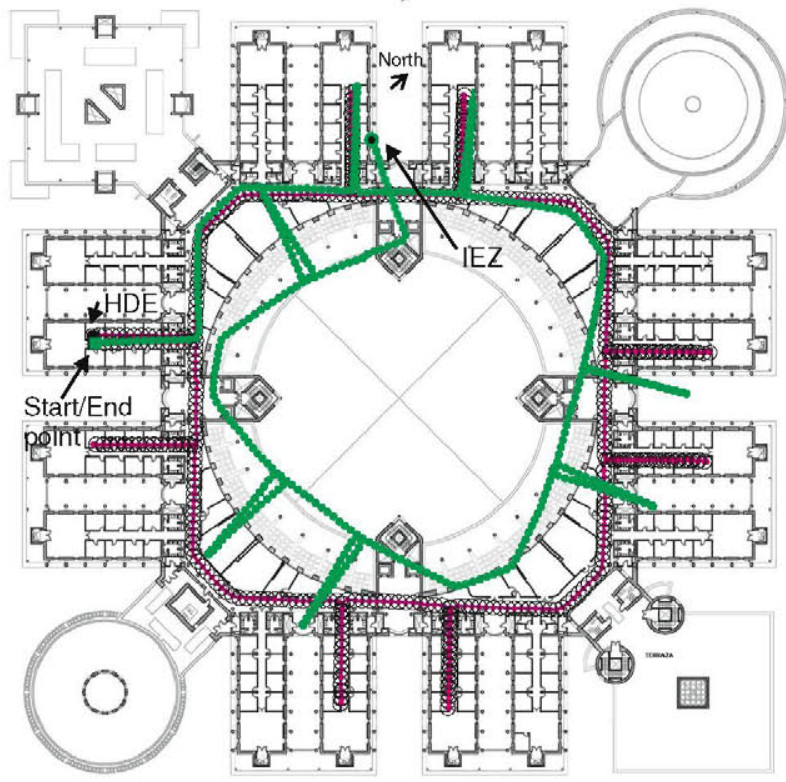

b)

Fig. 2. a) Building with a complex layout: The Engineering School of the University of Alcalá-de-Henares (UAH) in Spain. b) PDR trajectory in the third floor of the building above (an ideal floor for HDE navigation). In green color, the INS-based IEZ method (no magnetometers) [4]. The HDE solution $\left(\Delta=45^{\circ}\right)$ is represented in magenta color, with black circles at the detected steps where the HDE correction is performed.

\section{MAGNETIC COMPASSING INDOORS: LIMITS AND BENEFITS}

\section{A. Limitations of Magnetic Compassing}

The Earth Magnetic field has a known and constant magnitude and direction (vector) at a particular region on the Earth (see the International Geomagnetic Reference Field (IGRF) [7] for details). This magnitude can be measured with a 3-axis magnetometer, and it should be constant if a user wearing the sensor is moving along a non magneticallyperturbed region. However, in practice most common indoors environments are affected by magnetic perturbations that causes a significant deformation of the Earth magnetic field. If an electronic compass is directly used to obtain the orientation of the person while walking, then a low quality PDR trajectory

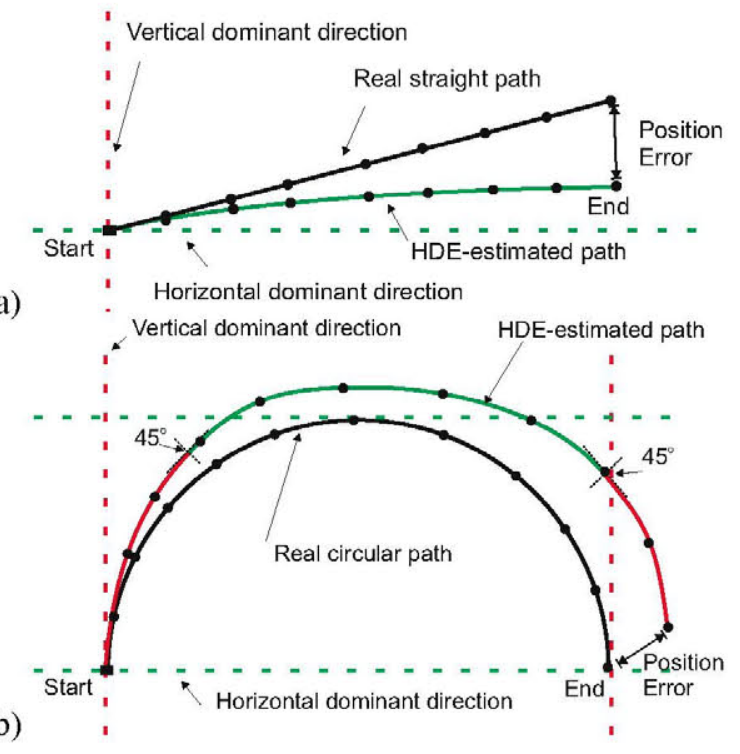

Fig. 3. Positioning error caused by the corrections of the original HDE method for: a) a straight path along a non-principal direction, and b) for a circular trajectory. This diagram only uses vertical and horizontal directions, i.e. $\Delta=90^{\circ}$. The color of the HDE-estimated path represents the building dominant direction to which the HDE correction is applied (red for vertical, and green for horizontal).

is estimated. Figure 4 shows an example of these facts, note the significant change in the magnetic field magnitude (a), the non-reliable yaw angle estimation (b), and the highly deformed trajectory for a real straight trajectory along a 60-meter-long corridor(c).

In view of these evidences, many authors have declared that the Earth magnetic field is not useful indoors [8], so they better relay on: higher quality IMUs (also bulkier and more costly), other external sensors (Local Positioning Systems, also known as LPS [9], [10]), or some heuristics (e.g. HDE, Mapmatching) in order to avoid the use of magnetometers. We believe that the magnetometric information, although somehow chaotic, provides some useful information (explained in next subsection) that could be used to improve PDR results.

\section{B. Benefits: Finding useful information in magnetic Yaw}

In order to explore the potentially useful information in the chaotic magnetometer readings, we performed several indoor walking experiments in three different buildings: University of Alcalá-de-Henares (UAH), University of Valladolid (UVa) and Center for Automation and Robotics (CAR-CSIC). Since the conclusions that we obtained were similar for each of the three buildings, next we will present only the experimental data corresponding to the UAH building. This is because UAH building interest us the most for the objectives of this paper i.e. it is a complex building with curved paths and 45-degreesoriented corridors.

At UAH building we recorded several paths along different corridors with diverse orientations for a total of about $3 \mathrm{~km}$ and 2130 user steps. The IMU was installed on the foot of the person and the measurements were made once per 


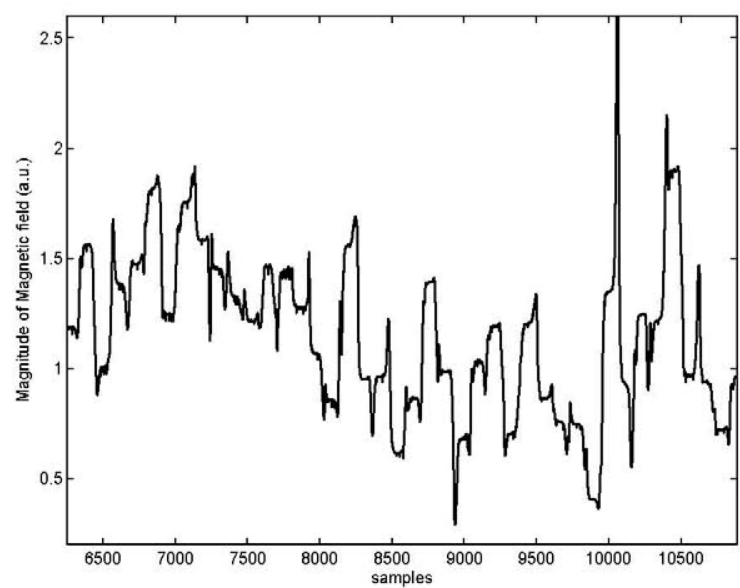

(a)

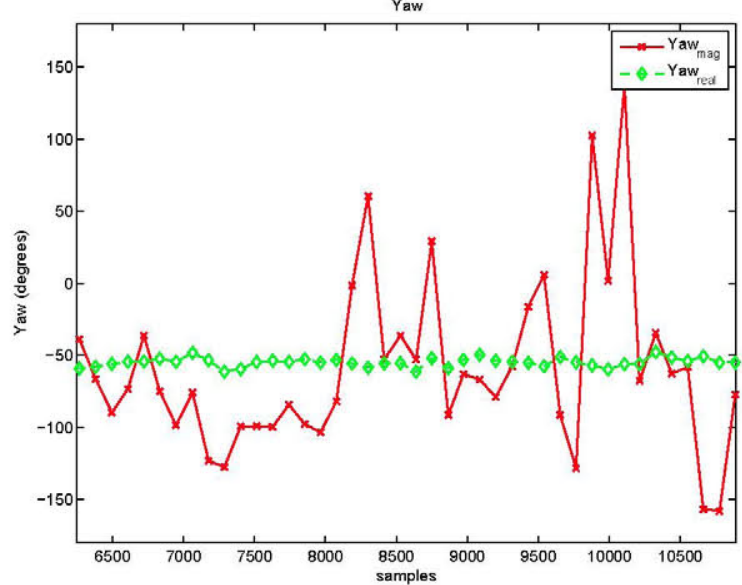

(b)

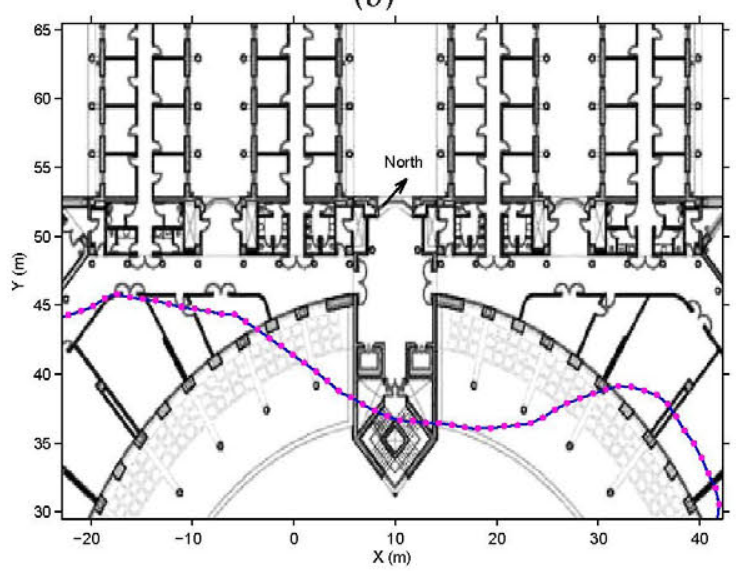

(c)

Fig. 4. Indoor Magnetic Compassing along a straight 60-meter-long corridor. (a) Magnitude of magnetic field captured with the Mti-Xsens sensor mounted on the foot of a person. (b) The compass-based Yaw angle estimation at each foot stance detection (red line) and the corresponding yaw reference (green line). (c) Estimated low-quality trajectory along the 60-meter-long corridor using IEZ algorithm ([4]) with magnetometer compassing.

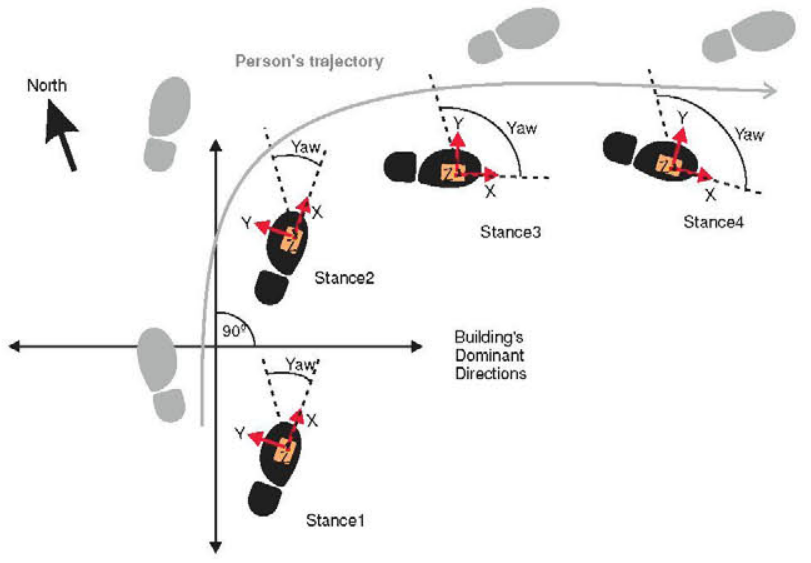

Fig. 5. A person's trajectory along some building's dominant directions. The IMU is installed on the right foot (black footstep; the gray footstep corresponds to the left foot). The Yaw angle of the sensor's X-axis with respect to the North is also annotated; this "X-axis Yaw angle" is one of the attitude parameters continuously estimated with the PDR algorithms. In Figure $4 \mathrm{~b}$ and in the upper graph of figure 6 some of these " $\mathrm{X}$-axis Yaw angles" are displayed.

each detected foot stance. Each measurement contains the horizontal Yaw angle of the sensor's X-axis with respect to the North (see Figure 5). This Yaw angle is computed from the magnetometer (Yawmag) as in [4]. Also, we have a reference Yaw angle or ground truth (Yaw real), which is deduced automatically from our PDR algorithms with the HDE heuristic enabled in order to keep the trajectory well aligned with respect to the dominant principal directions of the building [11]. Note that the yaw of the sensor's X-axis will not be necessarily aligned with the closest dominant directions of the building since it depends on how the IMU was installed on the foot and the typical orientation of the foot with respect to the direction of movement (this discrepancy is about 20 degrees in our experiments and has no effect on the conclusions obtained next).

The upper plot in Figure 6 shows the totality of yaw angles (Yawmag and Yaw ${ }_{\text {real }}$ ) recorded at the UAH building. The discrepancies (yaw error or $\Delta \psi$ ) between these two angles are plotted in the lower plot of Figure 6 as a black trace. It can be seen that the yaw error dispersion is significant, as expected, in any part of the tests. The important result is that the mean of all these yaw errors is almost zero ( -2.5 degrees) as is marked with the red line. Another important fact is that a simple average within a window of the last 100 yaw errors is also close to zero (blue plot at the bottom of Figure 6). This means that no significant systematic errors towards one preferred direction persists for more than 20-30 steps (as seen in the example of Figure $4 b$ ), that is, errors have a sign uniformly distributed. In summary, yaw orientation measured with a magnetometerbased compass has a significant dispersion but an approximate zero mean, so the compass can be very useful at a mid- or long-term scale, as we propose in this paper.

Additionally, the distribution of the yaw error is mainly Gaussian as can be deduced from the upper histogram in Figure 7. This histogram has superposed the mean yaw error 

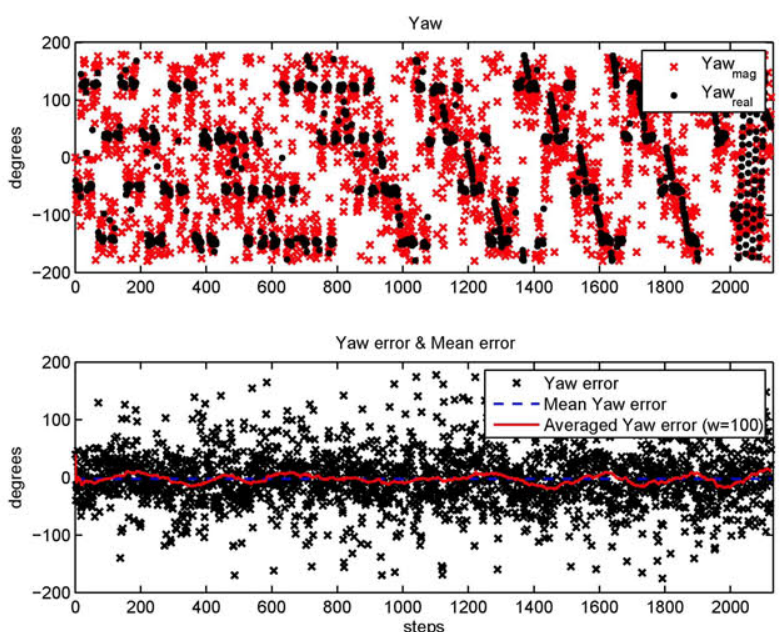

Fig. 6. Yaw angles captured for all UAH experiments that account for a tota of 2130 user steps. Above: The Yaw angle measured from the magnetometers (red crosses) and the real Yaw angle (black dots). Below: The difference between above values $\left(\Delta \psi=\right.$ Yawmag - Yaw $\left._{\text {real }}\right)$, i.e. the Yaw error that is plotted with black crosses. Also below, the average of all yaw errors (blue dashed line) and the average of the last 100 yaw errors (red continuous line).
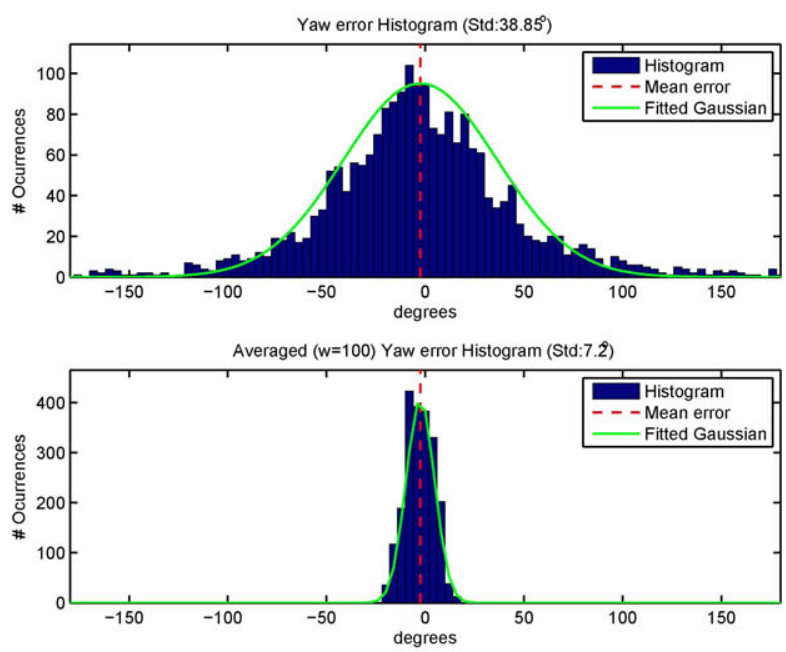

Fig. 7. Histogram of all Yaw errors $(\Delta \psi)$. The mean value is marked with a vertical dashed red line, and a fitted Gaussian is superposed in green color.

value (-2.5 degrees) and a Gaussian function with an standard deviation of 38.8 degrees which models most measured yaw errors, except some outliers above \pm 120 degrees that only represent a $2.8 \%$ of the total measured angles. The averaged of the yaw errors using a window of size 100 (lower histogram of Figure 7) can also be fitted with a Gaussian in this case with an standard deviation of $7.2^{\circ}$ and maximum discrepancies of about 20 degrees, which is good enough for some of our goals: perform magnetically-supervised dominant direction assignments.

As a complementary visualization of the obtained compassbased yaw angles we show in Figure 8 a three dimensional histogram to explore the relationship between the real and magnetometer-based yaw angles. In this $3 \mathrm{D}$ histogram we

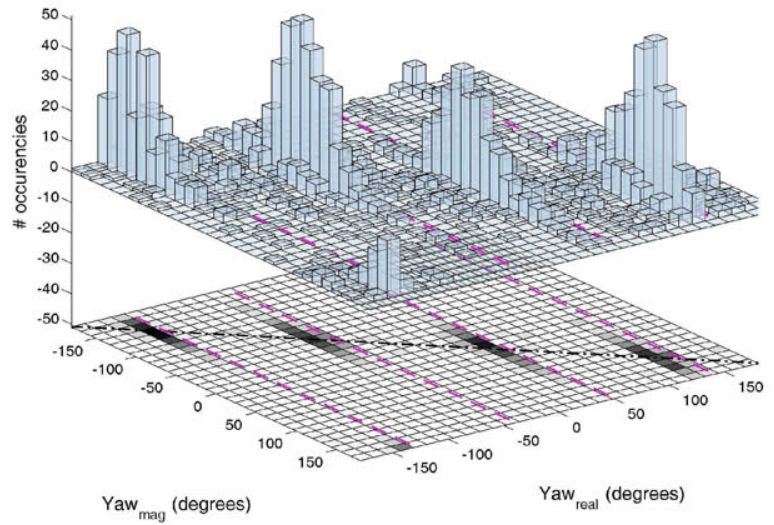

Fig. 8. Histogram in three-dimensions in order to visualize the correlation between the true orientations ( Yaw $_{\text {real }}$ ) and the yaw angles obtained from the compass (Yawmag). A projection of the histogram is shown at the bottom in order to see the correlation between both parameters (ideal relation is represented with the dashed black diagonal line). The building's dominant directions are marked, as a reference, with four parallel dashed magenta lines.

can observe how the compass-based yaw (Yawmag) are in correlation with the real yaws (the ideal diagonal correlation line is added as a reference dotted line below the histogram). The main four dominant directions of the building are superposed as magenta straight lines at angles:-130, -40 , 50 and 140 degrees. Note that the histogram is composed basically of measurements performed along these dominant directions, but also some data were captured at other angles since the tests included walk along 45-degrees directions and curved trajectories.

Finally, we split the data in four groups containing only the yaw errors for each one of the main four dominant directions. In Figure 9, we can visualize an histogram for each group that shows the clear separation that exists between the measurements models (Gaussian functions) for each of the four directions. Note that the dominant directions of the building (vertical lines in magenta color) are 20 degrees shifted to the right from the mean of the Gaussians; this is just the systematic difference between the X-axis of the IMU at foot stance and the orientation of the trajectory path defined by the building's dominant directions. Statistically it is clear that the real dominant direction of walk could be deduced from these Gaussian measurement models using Bayessian estimation and hypothesis testing methods. Our approach to assist in the dominant direction correction method will be based on the above observed information although it will be implemented in a much more straight forward manner, as will be presented in next section.

\section{THE PROPOSED MIHDE METHOD}

The proposed MiHDE method represents an improvement over the original HDE implementation presented by Borenstein et al. [1] and Abdulrahim et al. [2]. Additionally, it is also 

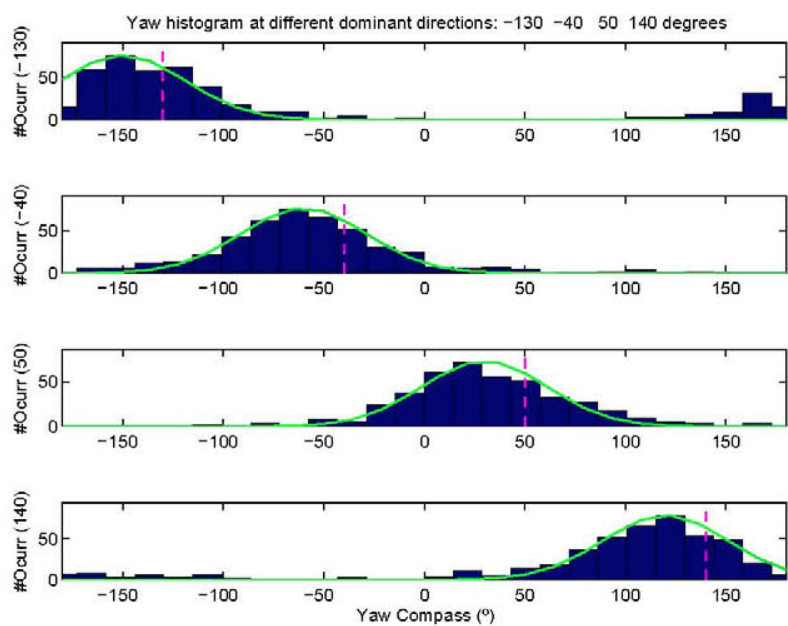

Fig. 9. Histograms of estimated compass-based yaw for each separated dominant direction of the building. A Gaussian is fitted to this histograms with a 38.8 degree standard deviation. The magenta dashed vertical lines represent the dominant directions of the building.

an extension over a previous work presented by the authors of this paper (Jiménez et al.) [11]; this former method was called iHDE and did not used the magnetometer information to help in the selection of the correct dominant direction. Next subsections will detail the different approaches included in MiHDE method, some of which are similar to the previous iHDE proposal.

\section{A. The IEZ Framework for pedestrian navigation}

We use the foot-mounted IMU-based PDR algorithm proposed by Foxlin [3] and later refined by Jiménez et al. [4]. This PDR method was termed as IEZ [4]; it stands for INSEKF-ZUPT i.e. a PDR algorithm that uses INS mechanization, an Extended Kalman Filter (EKF) and Zero-Velocity Updates (ZUPT). Fig. 10 represents a block diagram of the complete IEZ PDR method (only the white color boxes, since the lightgray color blocks corresponds to the extension proposed and explained later along this paper).

The basic IEZ PDR approach assumes that an IMU is installed on the foot of a person. An inertial navigation system (INS) algorithm is executed to integrate the accelerometer readings into velocity and them into position, also the gyroscope angular rate readings are integrated to obtain the attitude of the IMU sensor (i.e. Roll, Pitch and Yaw). Since performing a direct INS processing using low-performance IMU accumulates positioning and attitude error very quickly (severe drift), then it is mandatory to apply some corrections to refine the INS output. The most helpful correction is the Zero Velocity Update (ZUPT) which is used every time the foot is motion-less (stance phase) and consist in correcting the INS-estimated velocity with the "known" velocity of the foot at stance (zero velocity). This is a very effective way to reset the error in velocity of the INS. Another useful correction is applied when the person does not walk (still), it is called Zero Angular Rate Update (ZARU) because it assumes that at a still

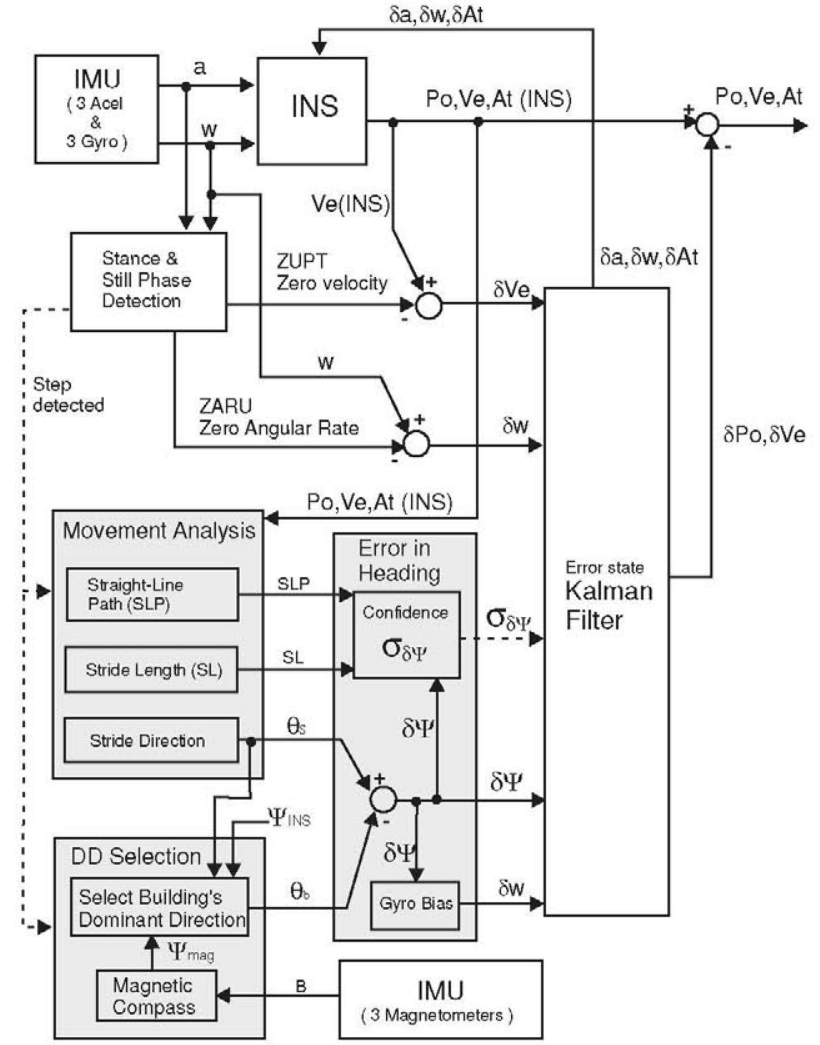

Fig. 10. The proposed MiHDE method for improved heading error elimination. It is implemented over the IEZ PDR framework [4].

condition the values measured from the gyroscope correspond basically to the sensor's biases. ZARU is an effective way to help in the estimation of the gyroscope biases, and it causes a better attitude estimation and specially a lower drift in orientation.

In order to optimally perform the measurement updates, the IEZ PDR algorithm uses an Extended Kalman Filter (EKF) which takes into account the uncertainties of estimations and measurements. The measurements used in the filter are velocity errors and gyroscope bias, using a Kalman filter with error states has benefits since the measurement model is linear and the observability of some states is improved. The IEZ PDR approach works with a 15 -element error state vector: $\mathbf{X}=\left[\delta \mathrm{At}, \delta \omega^{b}, \delta \mathrm{Po}, \delta \mathrm{Ve}, \delta \mathrm{a}^{b}\right]$. This vector contains the estimated bias of accelerometers and gyroscopes $\left(\delta \mathrm{a}^{b}\right.$ and $\delta \omega^{b}$, respectively), as well as, the $3 \mathrm{D}$ errors in attitude ( $\left.\delta \mathrm{At}\right)$, position $(\delta \mathrm{Po})$, and velocity $(\delta \mathrm{Ve})$.

Next subsections detail the extensions added to the basic IEZ PDR method to create the proposed MiHDE approach. The additional blocks in Fig. 10 (light-gray color blocks) represents the extra processing blocks for the MiHDE implementation that includes a "movement analysis" processing block, a "Dominant Direction (DD) selection" box, and an "error in heading" estimation block. 


\section{B. Movement Analysis in MiHDE}

Our movement analysis block, analyzes the stride direction of the person when walking, the length of this stride and decides if the trajectory is straight. This information is used to design some attenuators that will restrict the corrections of HDE to only some sections of the path. They are needed to estimate the heading error and the confidence on that estimation.

1) Stride Direction: The heading or direction of movement of the pedestrian when walking is:

$$
\theta_{S}(k)=\arctan \left(\frac{\mathrm{Po}_{y}^{k}-\mathrm{Po}_{y}^{k-1}}{\mathrm{Po}_{x}^{k}-\mathrm{Po}_{x}^{k-1}}\right),
$$

where $k$ is the index of the $k$-th step, and $\mathrm{Po}=\left\{\mathrm{Po}_{x}, \mathrm{Po}_{y}, \mathrm{Po}_{z}\right\}$ are the estimated position coordinates.

2) Stride Length (SL): Knowing the Stride Length (SL),

$$
\mathrm{SL}(k)=\sqrt{\left(\mathrm{Po}_{x}^{k}-\mathrm{Po}_{x}^{k-1}\right)^{2}+\left(\mathrm{Po}_{y}^{k}-\mathrm{Po}_{y}^{k-1}\right)^{2}},
$$

a Step Size (SS) binary attenuator is computed as

$$
\mathrm{SS}(k)=\left\{\begin{array}{ll}
1 & \mathrm{SL}(k)>\mathrm{Th}_{\mathrm{SL}} \\
0 & \text { Otherwise }
\end{array},\right.
$$

which will be later used to reject HDE corrections when walking with short steps. A threshold for the SL of 1 meter $\left(\mathrm{Th}_{\mathrm{SL}}=1 \mathrm{~m}\right)$ is used.

3) Straight Line Path (SLP): We decided to require at least five user strides with similar orientation in order to classify a trajectory as straight. We compute a binary Straight-Line Path (SLP) parameter as:

$$
\operatorname{SLP}(k)= \begin{cases}1 & \max \left(\left|\theta_{S}(j)-\operatorname{mean}\left(\theta_{S}(j)\right)\right|\right)<\operatorname{Th}_{\theta} \\ & \text { for } j=k: k-4 \\ 0 & \text { Otherwise }\end{cases}
$$

where $\mathrm{Th}_{\theta}$ is an angular threshold. SLP is used to deactivate the perturbing HDE corrections at curved paths.

\section{Magnetically-aided Dominant Direction Selection}

In order to compute the correct Dominant Direction, $\theta_{b}$ (the ouput of the "DD Selection" block in Figure 10), we first compute the Yaw orientation discrepancy $\Delta \psi$ (eq.5), then its average $\overline{\Delta \psi}$ (eq.6), after that the magnectically-aided direction of walk $\theta_{S}^{\mathrm{mag}}$ (eq.7), and finally we obtain the desired correct Dominant Direction $\theta_{b}$ (eq.8), which is a magnetically-assisted method to choose the correct DD.

First, the discrepancy in the Yaw orientation of the IMU $\mathrm{X}$-axis is computed as the difference between the magnetic compass output ( $\psi_{\mathrm{mag}}$ ), computed as in [4], and the PDRINS output ( $\left.\psi_{\mathrm{INS}}\right)$ for each foot stance detection:

$$
\Delta \psi(k)=\psi_{\operatorname{mag}}(k)-\psi_{\mathrm{INS}}(k) .
$$

As this Yaw error $(\Delta \psi(k))$ is very noisy (see the bottom graph in Figure 6) we do not use this information directly, in fact we use the average of the yaw errors in a large window of size 100 (i.e. the mean of the errors in the last one hundred steps).

$$
\overline{\Delta \psi}(k)=\frac{1}{100} \cdot \sum_{i=k-99}^{k} \Delta \psi(i)
$$

where $k$ is the index of the current $k$-th step.

It is important to remark that this mean error angle $(\overline{\Delta \psi})$ should be close to zero whenever the trajectory and orientation of the PDR-INS output is accurate. This value will start to increase or decrease slowly if the drift in heading is important due to significant non-compensated gyro biases or a long time without MiHDE corrections because, e.g., of continuos curved trajectories.

The magnetically-aided direction of walk (heading of the trajectory, $\left.\theta_{S}^{\operatorname{mag}}(k)\right)$ is computed as the addition of the stride direction $\left(\theta_{S}(k)\right.$ in eq. 1) and the mean error angle $(\overline{\Delta \psi}(k))$

$$
\theta_{S}^{\operatorname{mag}}(k)=\theta_{S}(k)+\overline{\Delta \psi}(k) .
$$

Note in these equations that $\psi$ angles refer to the IMU's X-axis yaw, and $\theta$ angular notation is used also to measure yaw but in this case of trajectories and building dominant directions (in any case they use the same local navigation frame aligned with Geographic North, West and Up)

Finally, the selection of the dominant direction (DD) that best fits the current trajectory $\left(\theta_{b}(k)\right)$ takes into account the mid-term magnetic yaw information and it is computed as:

$$
\theta_{b}(k)=\arg \min (\theta) \quad\left|\{\theta \mid \mathrm{DD}\}-\theta_{S}^{\operatorname{mag}}(k)\right|,
$$

where the different dominant directions (DD), for example in the UAH building have these 8 values: DD = $\{-175,-130,-85,-40,5,50,95,140\}$ (in degrees). This method of computing $\theta_{b}(k)$ has the potential to select the correct DD even when the PDR orientation estimation is wrong (possible after a very long period without MiHDE observations).

\section{Estimating the error in heading in MiHDE}

The error in heading is computed as a direct substraction between the stride direction $\theta_{S}(k)$ at step $k$, and the closest dominant direction of the building $\theta_{b}(k)$, as:

$$
\delta \psi(k)=\theta_{S}(k)-\theta_{b}(k) .
$$

This is the error in heading that is fed into the EKF for a subsequent heading correction and an internal gyro bias estimation.

\section{E. Confidence of the error in heading}

We define the following expression for the standard deviation of the error in heading $\left(\sigma_{\delta \psi}\right)$, so as to make the MiHDE heading correction adaptive with each kind of motion:

$$
\sigma_{\delta \psi}(k)=\frac{\sigma_{\mathrm{HDE}}}{\operatorname{SLP}(k) \cdot \mathrm{SS}(k) \cdot e^{-5|\delta \psi(k)+\overline{\Delta \psi}(k)| / \Delta}} .
$$


The value of $\sigma_{\mathrm{HDE}}$ is selected as a trade-off to give a reasonable confidence about the HDE-based yaw corrections, in our case is selected as 0.1 radians. The SS and SLP terms are the binary values computed in equations 3 and 4, they mean that in case of a Straight Line Path with long-enough steps, then the heuristic is applied, otherwise it value is zero and therefore the $\sigma_{\delta \psi}$ is infinite (no confidence), i.e. no MiHDE correction is applied. The exponential term $\left(e^{-5 \mid \delta \psi+\overline{\Delta \psi} / \Delta}\right)$ is used to limit the correction from straight paths not too well aligned with the building's dominant directions. In summary, only straight well-aligned paths at a normal pace are basically used in MiHDE. This contrasts with the original HDE method that always applies corrections, even in curved trajectories, whenever the steps are long enough.

\section{F. Additional gyro bias estimation support}

In order to increase the observability of gyro biases during walk (note that Gyro biases are mainly well estimated during Still periods with ZARU, but these updates are not frequent in practice), we added a block that increase or decrease the current bias value whenever it is detected a systematic yaw error (e.g. $\delta \psi$ has a predominant sign). This method assumes that if an almost perfect straight trajectory is systematically curved to the right or Clock Wise direction ( $\mathrm{CW}$ drift), then the bias of the vertical gyroscope is over-estimated, and viceversa trajectories that curve to the left or Counter Clock Wise direction (CCW drift) are caused by under-estimated biases. So in order to correct the bias, incremental small quantities are added or subtracted to the current bias estimates to attenuate long-term systematic drifts in heading.

$$
\delta w(k)=\delta w(k)^{-}+\Delta w \cdot \operatorname{sign}\left\{R_{n}^{b}(k)^{-} *(0,0, \delta \psi(k))\right\}
$$

where $\Delta w$ is an arbitrary constant that represents the small quantities added or subtracted to the gyro biases. The term $\delta w(k)^{-}$is the a priori gyro bias vector ( 3 components in $\mathrm{x}, \mathrm{y}$ and $\mathrm{z}$ axis), $R_{n}^{b}(k)^{-}$is the a priori rotation matrix that transforms a vector from the navigation frame (Noth-West-Up) to the body sensor frame, and the sign function takes a +1 for positive values, and -1 for negative ones. The confidence that we have on this correction is dependent on the confidence on the yaw correction ( $\sigma_{\delta \psi}$, in eq. 10), so corrections in gyros biases are only activated when MiHDE is active (long steps, straight trajectories, paths aligned to dominant directions,...).

\section{EXPERIMENTAL EVALUATION}

For the evaluation of the proposed MiHDE method, and for comparing it to the IEZ and HDE methods, we use both, synthetically generated IMU signal with a ground-truth, and also real experiments performed at a building using a foot mounted IMU.

\section{A. Evaluation using a synthetically generated IMU signal with a ground-truth}

We have employed several synthetically-generated IMU signals using the methodology proposed in [12]. Each generated IMU signal has a ground-truth of the position (as well as attitude and velocity) for every sample in the simulated trajectory. The ideal IMU signal sampled at $100 \mathrm{~Hz}$, was contaminated with a known constant bias at the gyroscopes $(0.01,0.006$ and $0.003 \mathrm{rad} / \mathrm{s}$ for axes $\mathrm{x}, \mathrm{y}$ and $\mathrm{z}$, respectively). All trajectories generated have an initial and final interval where the IMU is motionless, in particular the simulation considers that the person (the foot) is still 10 seconds before starting to move, and also just after ending the trajectory for another 10 seconds.

A square trajectory repeated twice was generated as an "easy" one satisfying very well the HDE assumptions (moving along two principal directions: North-South or East-West; i.e. $\Delta=90^{\circ}$ ). In this case the IEZ method is expected to accumulate drift in heading, but HDE and MiHDE should clearly get advantage of the dominant directions corrections to eliminate the drift. Results are shown in Fig. 11.

We observe in Fig. 11a that the IEZ solution has some drift in yaw, as expected, however this drift is not so damaging since the ZARU correction of IEZ during the initial 10 seconds interval (first 1000 samples) allows the system to partially estimate the gyro biases. During the motion there is no observability of yaw angle, so estimated biases do not improve, although the uncertainly in the covariance matrices of estimates grows. The final still phase achieves the correct estimation of gy ro biases. For the HDE method we observe in Fig. $11 \mathrm{~b}$ that yaw is observable and consequently the bias of gyroscopes. After $100 \mathrm{~s}$ of walk (10.000 samples) biases are well estimated. The 8 spikes in the bias plot corresponds to the 8 turns that slightly perturbs the estimations. The MiHDE method performs similarly to HDE as can be seen in Fig. 11c, but in this case no perturbations appear since during turns no corrections are applied. For this "ideal" type of trajectories both HDE and MiHDE method perform quite well eliminating the drift in heading.

A more challenging trajectory for $\mathrm{HDE}$ is evaluated as presented in Fig. 12. This trajectory consists of two straight line segments aligned with one of the dominant directions (west-east) at the beginning and end sections, and in the middle a straight-line segment $30^{\circ}$ degrees oblique from the dominant direction. The bias convergence in Fig. 12a for IEZ is similar to the case presented before in Fig. 13a. The middle segment is not correctly processed by HDE method, neither in the position estimation nor in the bias estimation. In fact the bias is wrongly estimated during this oblique path (samples from 2800 to 4400 ). When the path is again aligned with the dominant direction (samples 4400 to 6100 ) the bias is progressively recovered to the true value. The performance of MiHDE is improved simply by ignoring the yaw corrections during the non-aligned sections of walk, under this case it basically uses the previously computed biases.

Another challenging trajectory for $\mathrm{HDE}$ is evaluated as presented in Fig. 13. This trajectory consists of two straight line segments aligned with one of the dominant directions (west-east) at the beginning and end sections, and in the middle two iterations of a circular trajectory having a radius of 10 meters. The IEZ performs as usual, it is basically 

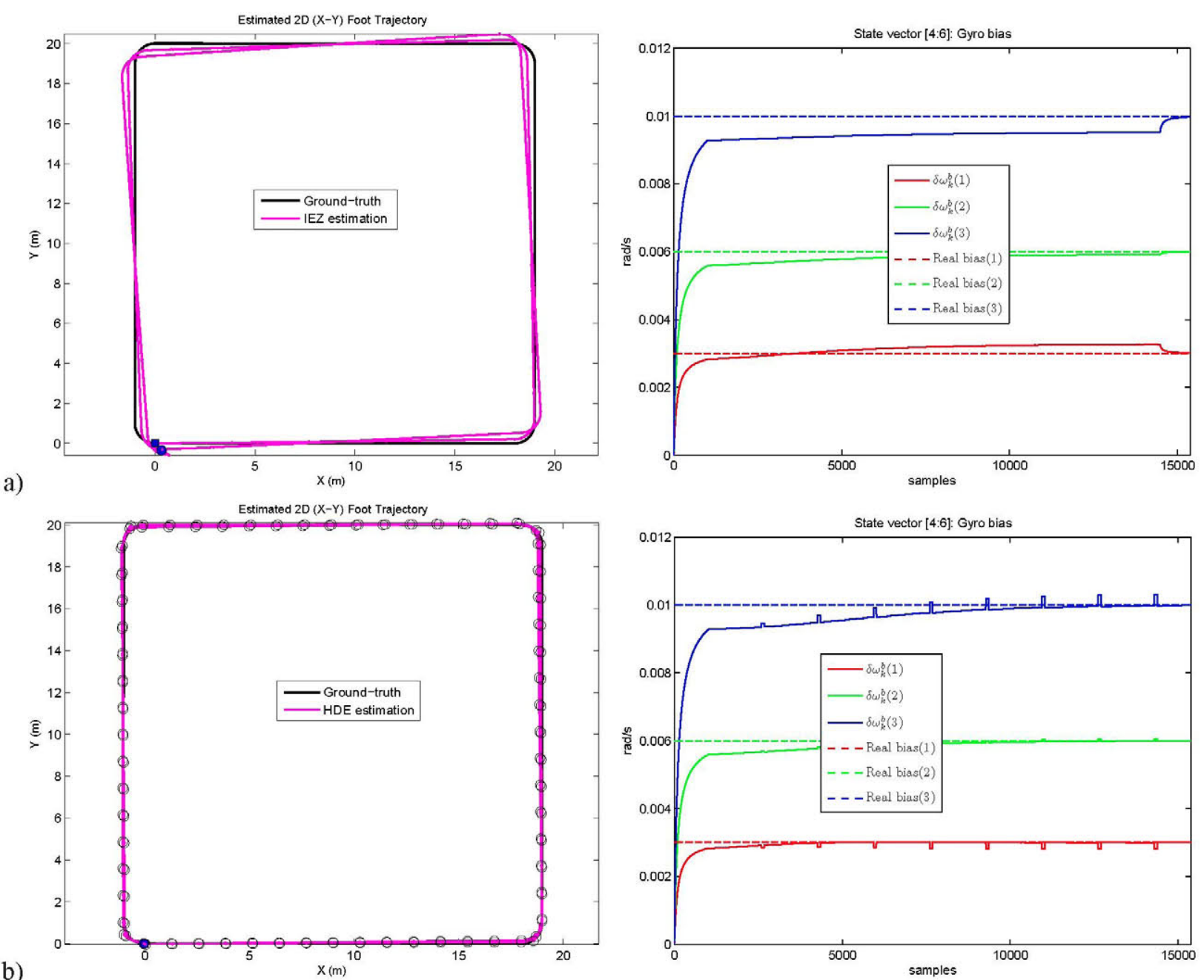

b)

Estimated $2 \mathrm{D}(\mathrm{X}-\mathrm{Y})$ Foot Trajectory
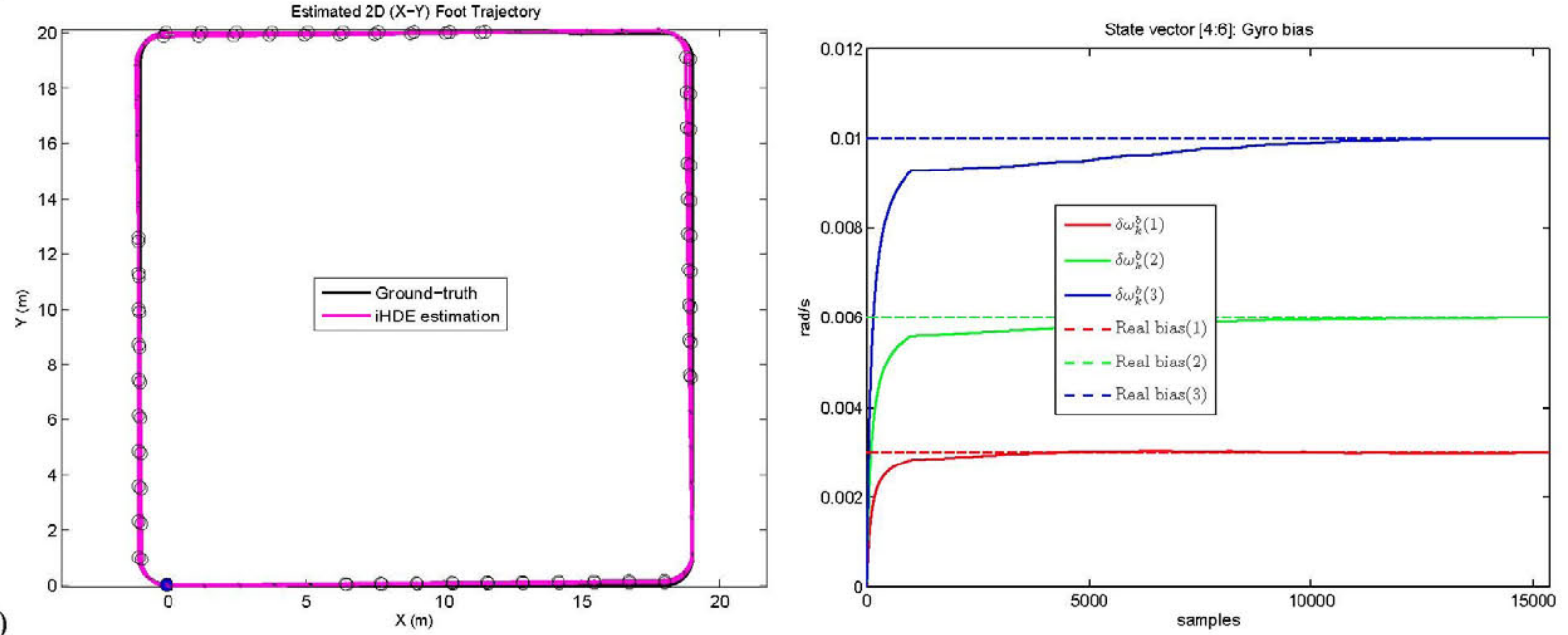

Fig. 11. Evaluation of algorithms using a synthetically-generated IMU signal that corresponds to a square trajectory repeated twice. a) Estimated trajectory using IEZ method (left), and the estimation of the biases of the 3-axes gyroscope (right); b) The same as before for HDE method; c) The same as before for MiHDE method. 

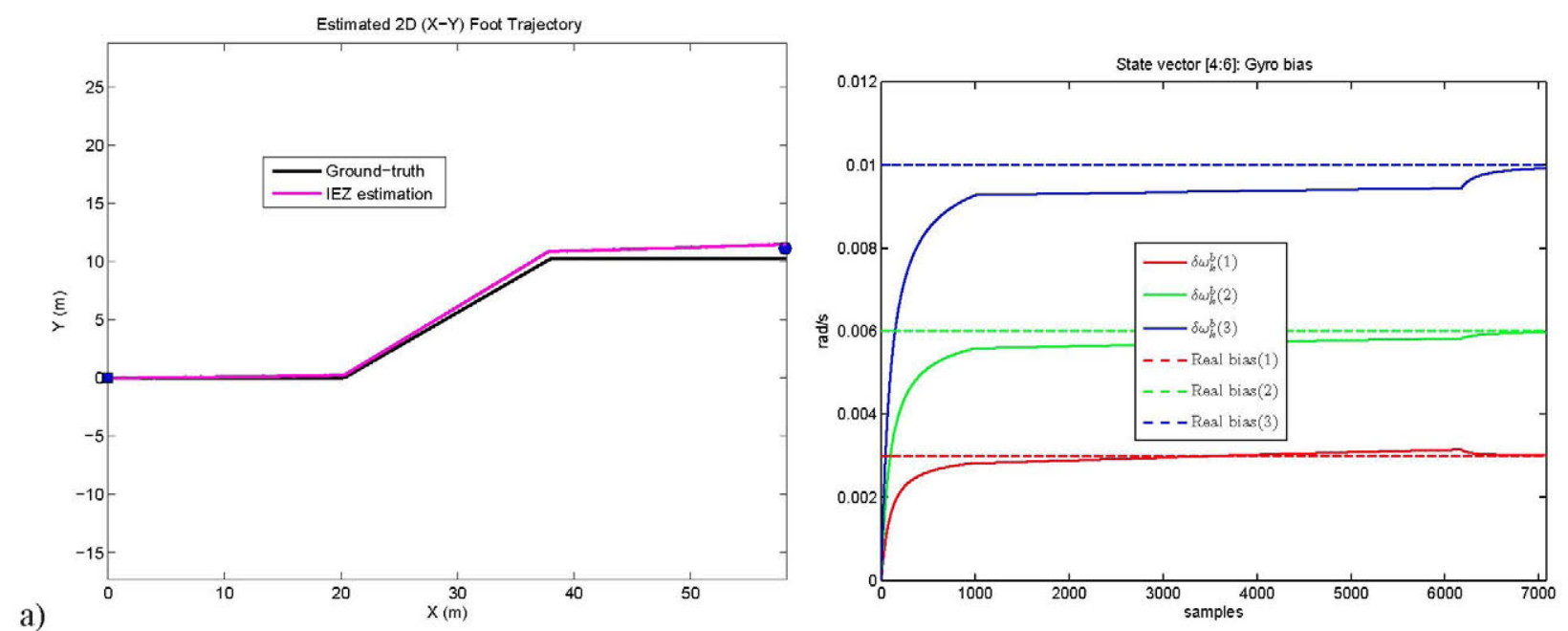

Estimated 2D $(X-Y)$ Foot Trajectory

b)
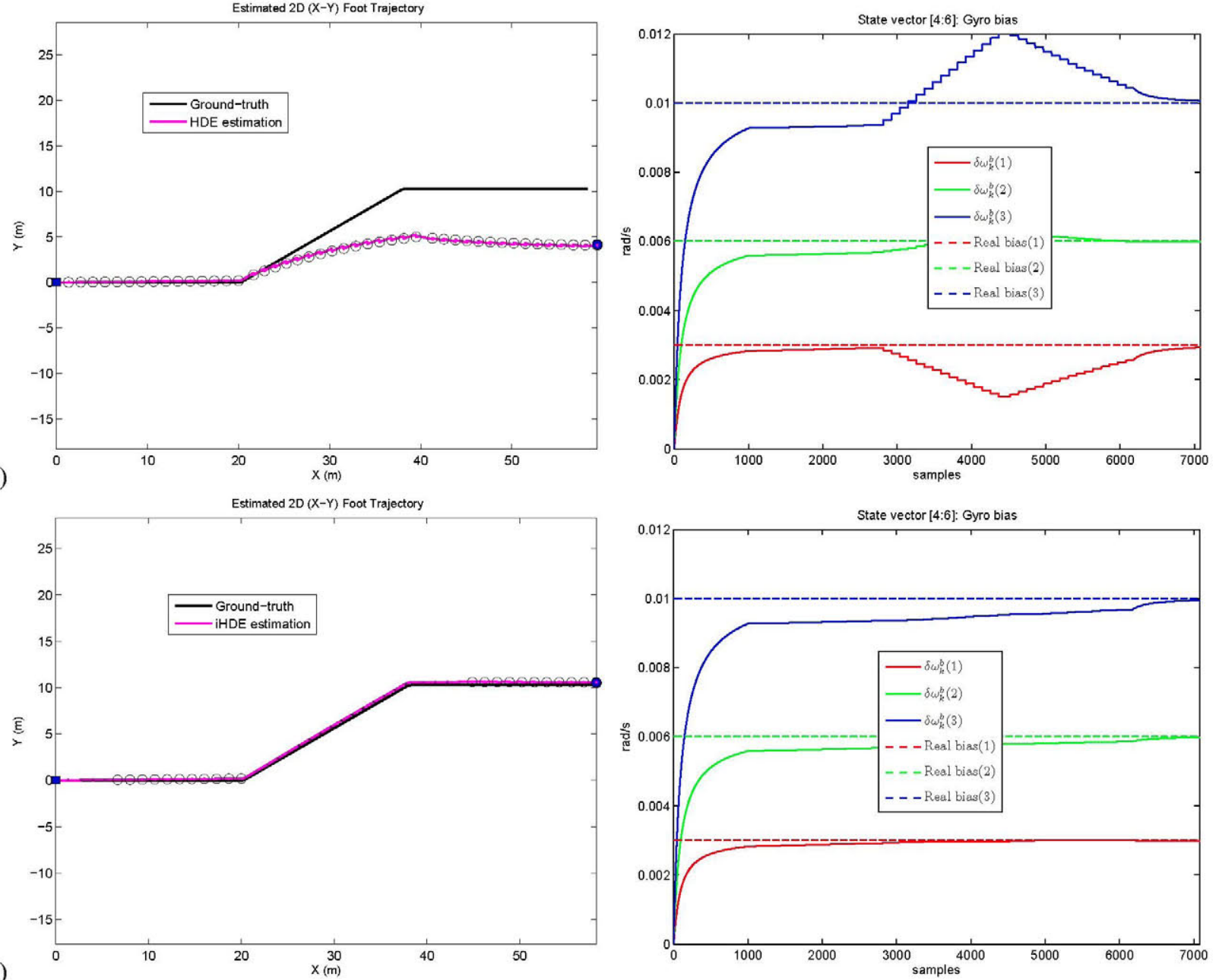

Fig. 12. Evaluation of algorithms using a synthetically-generated IMU signal that corresponds to two straight line segments aligned with one of the dominant directions and another $30^{\circ}$ oblique segment. a) Estimated trajectory using IEZ method (left), and the estimation of the biases of the 3-axes gyroscope (right); b) The same as before for HDE method; c) The same as before for MiHDE method. 
not dependent on the kind of trajectory, as it is observed in Fig. 13a. The degradation expected for HDE can be visualized in Fig. 13b, there is a deformation of the circular path shape and an error in the heading. This is caused by the alternative corrections in yaw on each two dominant directions (horizontal and vertical). The eight peaks in the bias estimations during the 2 circular paths corresponds to the 4 damaging correction along the directions in a single cycle: North-South, West-East, South-North, East-West. MiHDE on the contrary deactivates corrections during the circular path and consequently only accumulates a drift in heading similar to that of IEZ, but the positioning and heading error is corrected when walking again along a straight path at the end of the trajectory (see Fig. 13c).

\section{B. Evaluation using Real IMU signals recorded in a complex building}

Several tests were performed using a foot-mounted IMU (XSens Inc.) at the building shown in Fig. ?? $\left(\Delta=45^{\circ}\right)$.

1) Wide slightly-curved corridors: In the first floor of this building, there are wide curved corridors (see Fig.14a). We tested the HDE and the proposed MiHDE algorithms in these challenging conditions. The positioning results for a closed 460-meters-long path is shown in Fig. 14b and c. The damaging action of HDE is perceived mainly in the curved path in the east wing. MiHDE basically does not apply corrections on curves and achieves a slightly lower positioning error than HDE.

2) Circular Paths: Other results for circular paths are presented in Fig. 15. The damaging effect of HDE causes a position and orientation error when finishing the circular loops (e.g. after the 4 loops in Fig. 15 just before returning straight to the starting point). Other tests performed confirmed improvements of the MiHDE method over the HDE for routes including difficult trajectories (improvements of about $0.2 \%$ of TTD). In more "ideal" floors having long narrow corridors (like the third floor in Fig.2), the performance of HDE and $\mathrm{MiHDE}$ is quite similar, as expected.

\section{Evaluating the capability to avoid false dominant direction assignments}

In order to explore the capabilities of the MiHDE method to escape from false dominant direction matches, we have performed some trajectories that include very long circular paths. The idea is to let the PDR algorithm work without DD correction during a long time in order to accumulate a significant drift in heading. An accumulated heading error larger than 22.5 degrees will be enough to cause a wrong dominant direction match in HDE and HDE methods. With this kind of trajectories we should observe how the MiHDE is, on the contrary, able to detect this wrong situation and finally align with the true dominant direction.

We show in Figure 16 how the basic IEZ method accumulates a progressive drift in heading, as well as the HDE and iHDE algorithms perform well until the estimation reaches the region with the 20 consecutive circles. At the end of this repetitive circular path the orientation of HDE and HDE

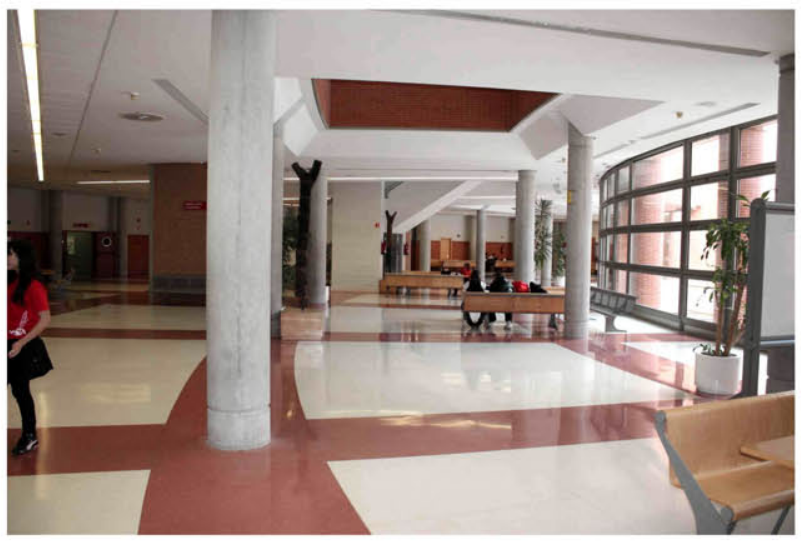

a)

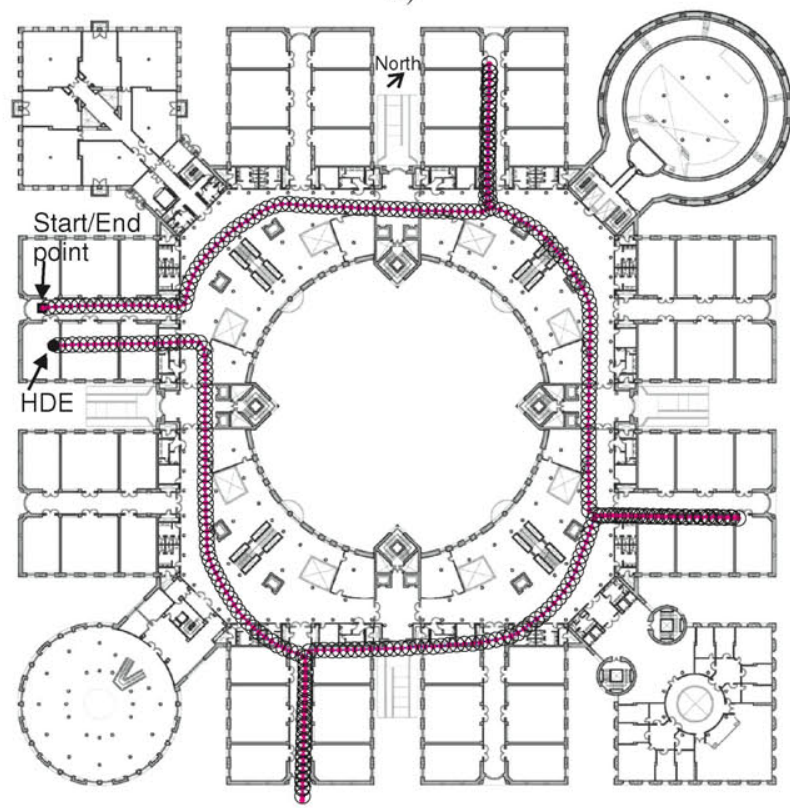

b)

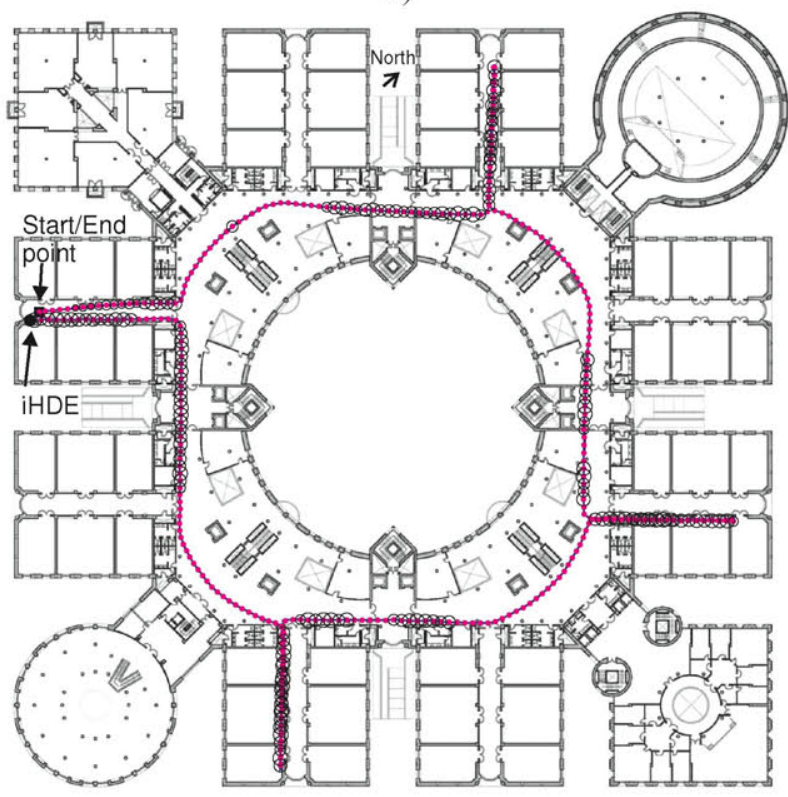

c)

Fig. 14. Tests in a floor with wide and curved corridors. a) Photo of the corridor, b) Estimation with HDE, c) Estimation with MiHDE. The black small circles in the path mark the HDE or MiHDE heading corrections. The size of these circles is inversely proportional to $\sigma_{\delta \psi}$. HDE is making corrections all the time with a constant $\sigma_{\delta \psi}=\sigma_{\mathrm{HDE}} / \mathrm{SS}$, however MiHDE corrects adaptively, mainly at well-aligned straight-line segments, using eq. 10 . 

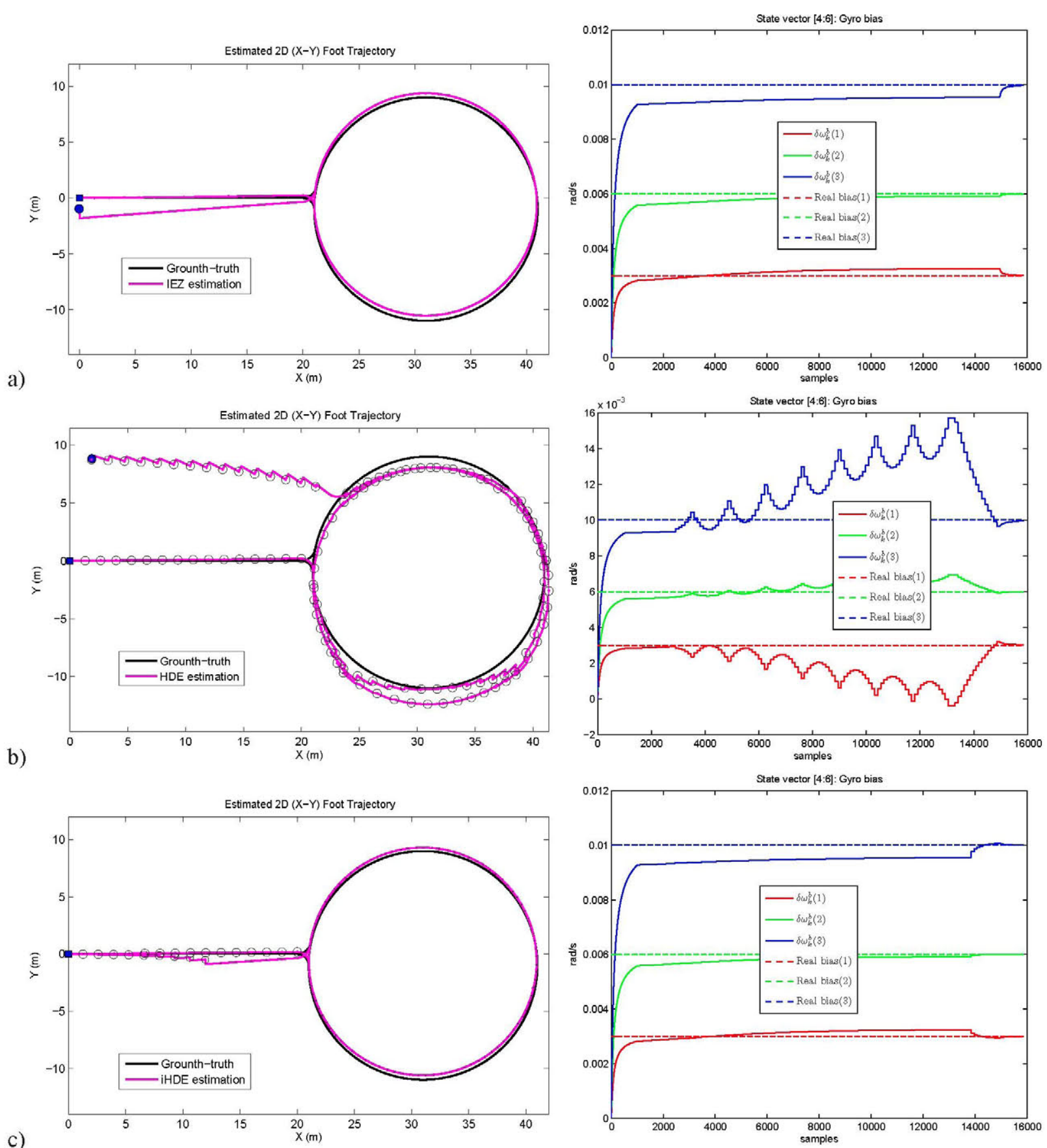

Fig. 13. Evaluation of algorithms using a synthetically-generated IMU signal that corresponds to two straight line segments aligned with one of the dominant directions and 2 circular paths in between having a radius of 10 meters. a) Estimated trajectory using IEZ method (left), and the estimation of the biases of the 3-axes gyroscope (right); b) The same as before for HDE method; c) The same as before for MiHDE method. 


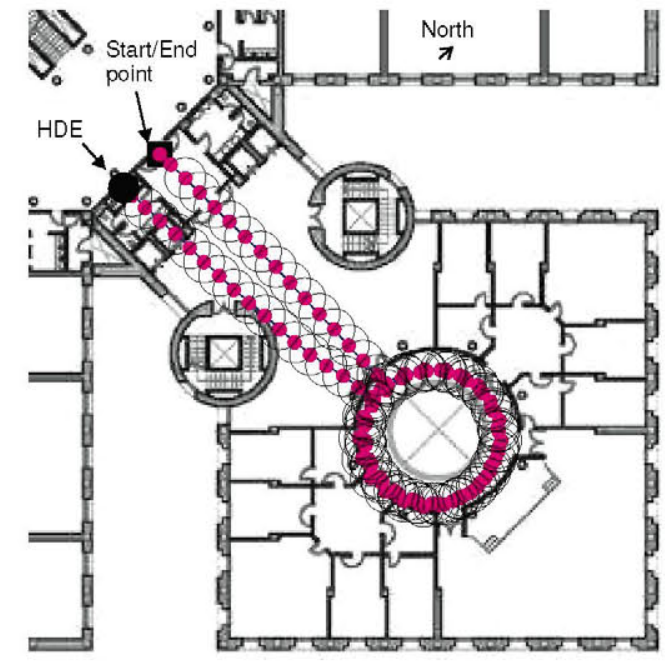

a)

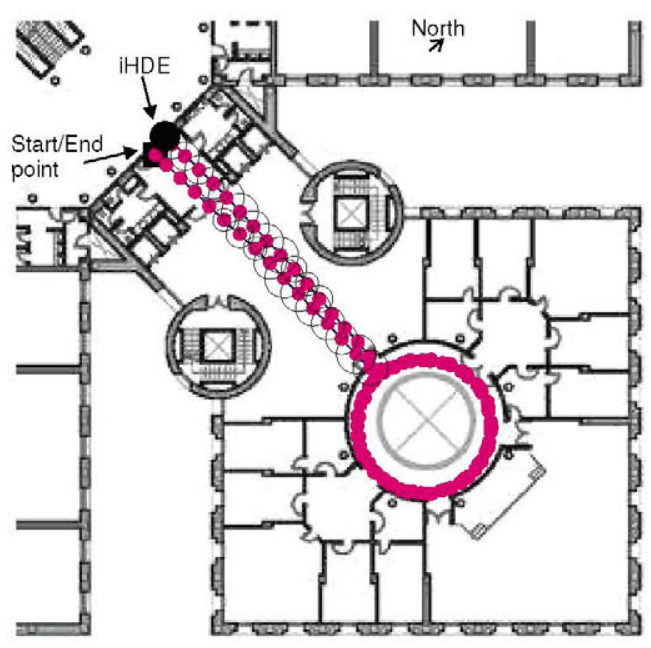

b)

Fig. 15. Test walking around a circular path 4 times (the starting and final path is straight at a $45^{\circ}$ dominant direction). a) HDE estimation, b) MiHDE estimation. The total route length is $146 \mathrm{~m}$.

algorithms is bad enough to be matched to a wrong dominant direction (the horizontal DD). We observe, however, that the MiHDE algorithm presented in this paper accumulates a similar bad alignment at the end of the circular paths, but it is able to distinguish the right dominant direction among the eight ones available. Therefore, the estimation is much better than in the other approaches.

\section{CONCLUSION}

We have analyzed the limitations of the HDE method, proposed a improved version (MiHDE), and tested both in challenging buildings. We confirm that the heuristic that uses the dominant's directions of the building is an extraordinary method to implement practical PDR indoor navigation solutions (with none or a minimum infrastructure), and it is a great alternative to pure compass-based navigation when magnetic disturbances are significant. We have demonstrated that even with severe magnetic perturbations a mid-term absolute heading correction is possible, so we put together the benefits from dominant directions in a building and the statistical zero-mean behavior of the magnetically-estimated heading.

\section{ACKNOWLEDGMENT}

The authors thank the financial support from projects LEMUR (TIN2009-14114-C04-03) and LAZARO (CSIC-PIE Ref.201150E039). Special thanks to J. Ureña and J.C. García from the Electronics Department of UAH for their help.

\section{REFERENCES}

[1] J. Borenstein and L. Ojeda, "Heuristic Drift Elimination for Personnel Tracking Systems," Journal of Navigation, vol. 63, pp. 591-606, Sept. 2010.

[2] K. Abdulrahim, C. Hide, T. Moore, and C. Hill, "Aiding MEMS IMU with building heading for indoor pedestrian navigation," in Ubiquitous Positioning Indoor Navigation and Location Based Service (UPINLBS), 2010, pp. 1-6, IEEE, 2010.

[3] E. Foxlin, "Pedestrian tracking with shoe-mounted inertial sensors," IEEE Computer Graphics and Applications, no. December, pp. 38 46, 2005.

[4] A. Jiménez, F. Seco, J. Prieto, and J. Guevara, "Indoor Pedestrian Navigation using an INS/EKF framework for Yaw Drift Reduction and a Foot-mounted IMU," in WPNC 2010: 7th Workshop on Positioning, Navigation and Communication, Dresden (Germany), vol. 10, pp. 135143,2010

[5] V. Renaudin, M. Afzal, and G, "New method for magnetometers based orientation estimation," in Position Location And Navigation Symposium, Indian Wells/Palm Springs, California USA, p. 9, 2010.

[6] M. H. Afzal, V. Renaudin, and G. Lachapelle, "Use of Earth's Magnetic Field for Mitigating Gyroscope Errors Regardless of Magnetic Perturbation," Sensors, vol. 11(12), pp. 11390-11414, 2011.

[7] C. C. Finlay, S. Maus, C. D. Beggan, T. N. Bondar, A. Chambodut, T. a. Chernova, A. Chulliat, V. P. Golovkov, B. Hamilton, M. Hamoudi, R. Holme, G. Hulot, W. Kuang, B. Langlais, V. Lesur, F. J. Lowes, H. Lühr, S. Macmillan, M. Mandea, S. McLean, C. Manoj, M. Menvielle, I. Michaelis, N. Olsen, J. Rauberg, M. Rother, T. J. Sabaka, A. Tangborn, L. Tø ffner Clausen, E. Thébault, a. W. P. Thomson, I. Wardinski, Z. Wei, and T. I. Zvereva, "International Geomagnetic Reference Field: the eleventh generation," Geophysical Journal International, vol. 183, pp. 1216-1230, Dec. 2010.

[8] P. Aggarwal, D. Thomas, L. Ojeda, and J. Borenstein, "Map matching and heuristic elimination of gyro drift for personal navigation systems in GPS-denied conditions," Measurement Science and Technology, vol. 22, p. 025205 , Feb. 2011.

[9] A. Jiménez, F. Seco Granja, J. C. Prieto Honorato, and J. Guevara Rosas, "Accurate Pedestrian Indoor Navigation by Tightly Coupling a Foot-mounted IMU and RFID Measurements," IEEE Transactions on Instrumentation and Measurement, vol. 61, no. 1, pp. 178 - 189, 2011.

[10] J. Hightower and G. Borriello, "Location Systems for Ubiquitous Computing," Computer, vol. 34, no. 8, pp. $57-66,2001$.

[11] A. Jiménez, F. Seco, F. Zampella, J. C. Prieto, and J. Guevara, "Improved Heuristic Drift Elimination ( $\mathrm{iHDE}$ ) for Pedestrian Navigation in Complex Buildings," in IPIN, no. September, pp. 21-23, 2011.

[12] F. J. Zampella, A. R. Jiménez, F. Seco, J. C. Prieto, and J. I. Guevara "Simulation of Foot-Mounted IMU Signals for the Evaluation of PDR Algorithms," in International Conference on Indoor Positioning and Indoor Navigation (IPIN), no. September, pp. 21-23, 2011. 


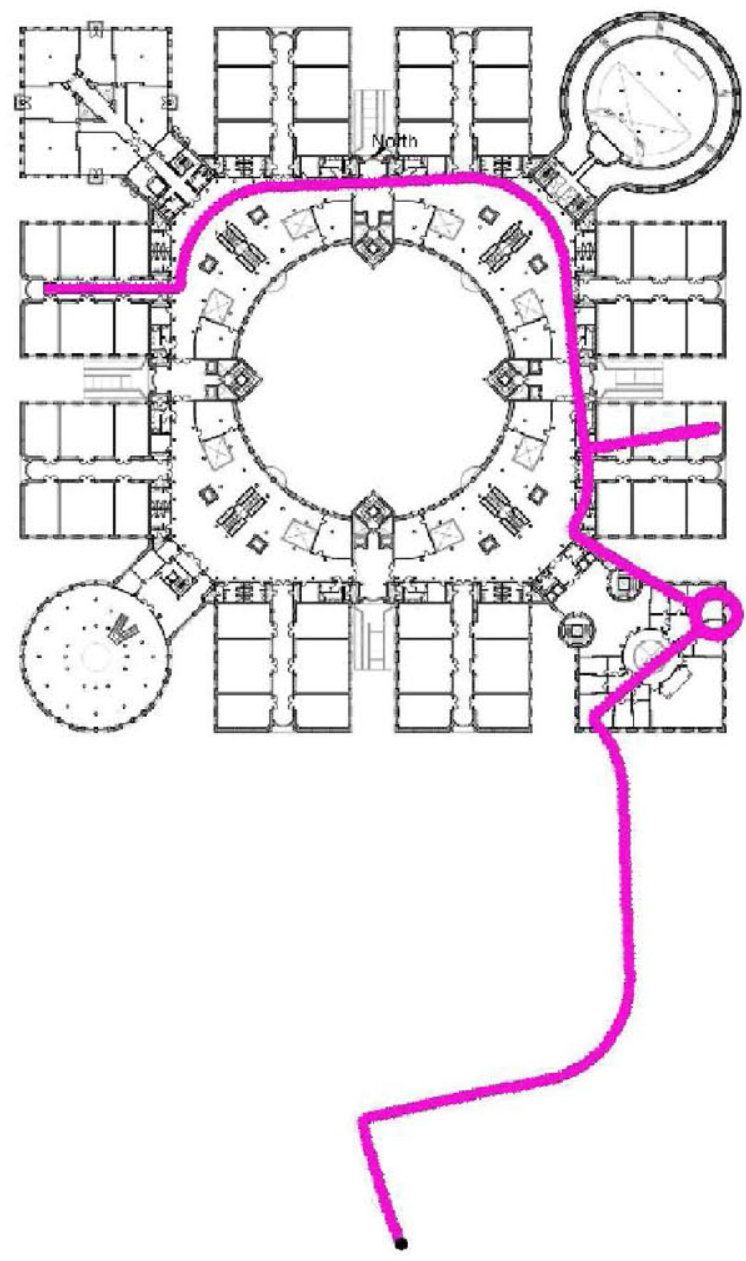

a)

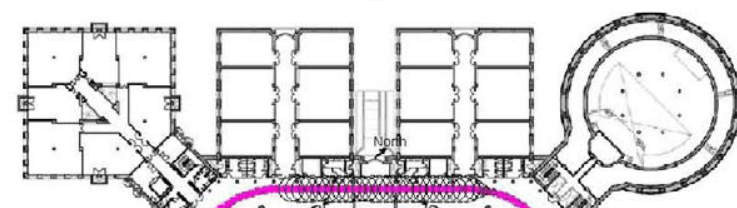

b)
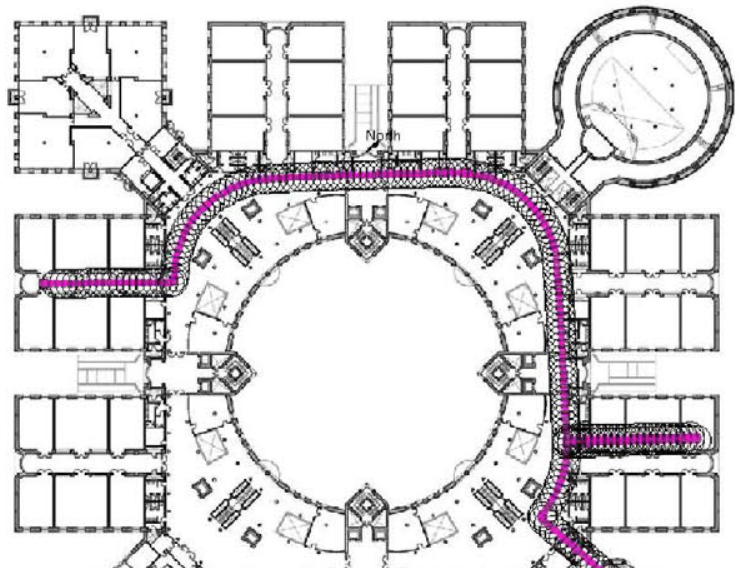

Q.
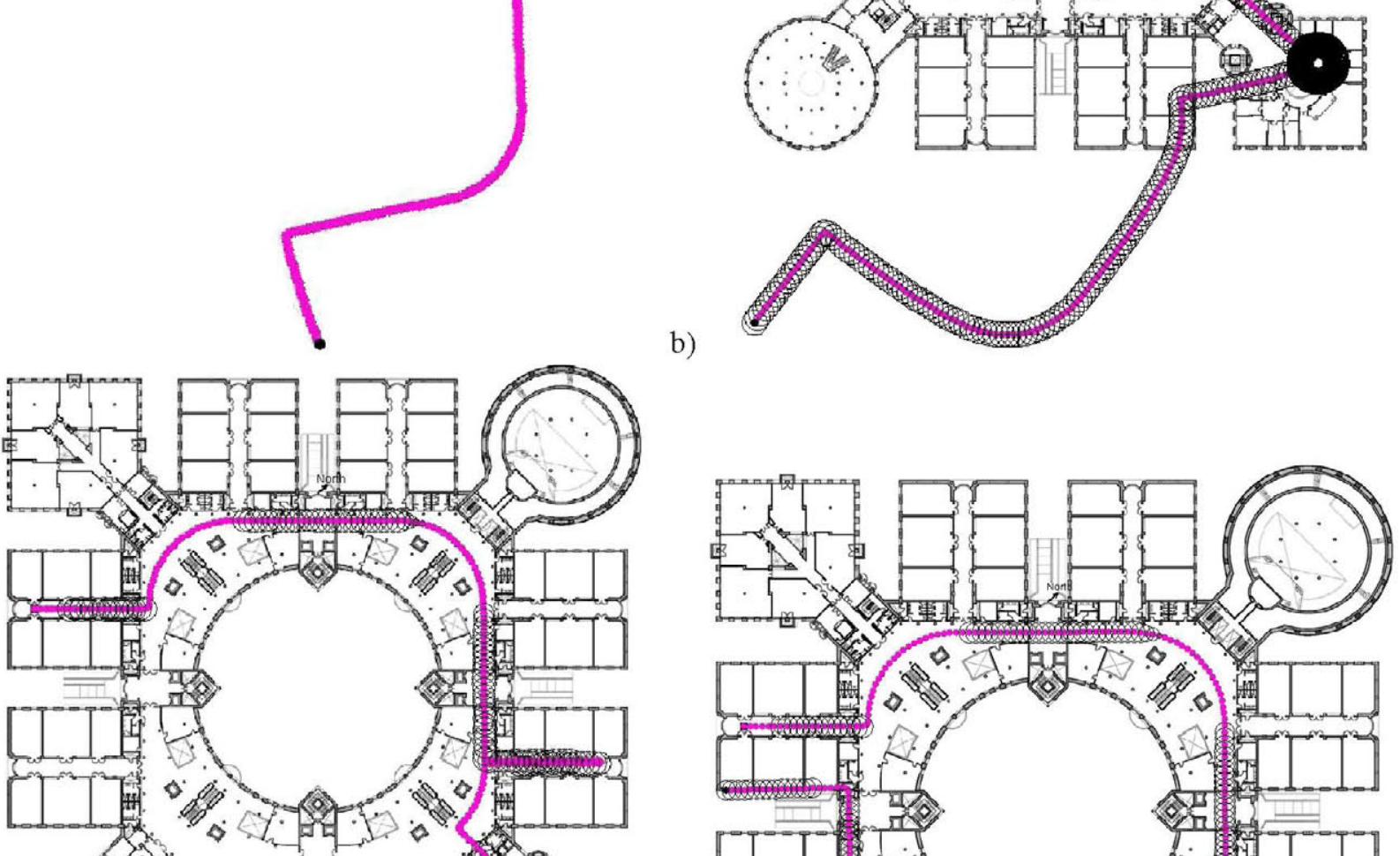

\section{(1)}

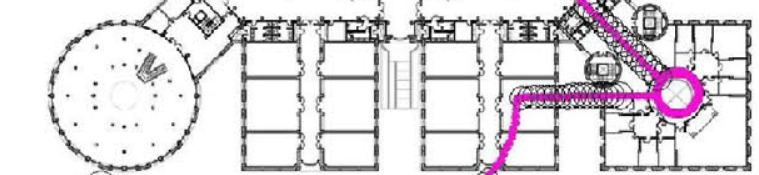

c)

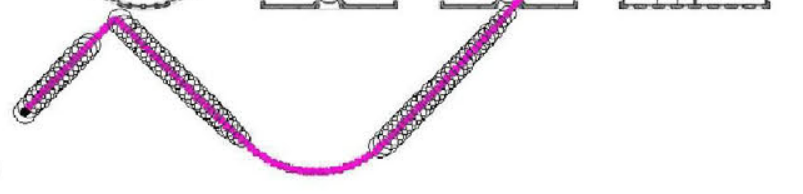

d)

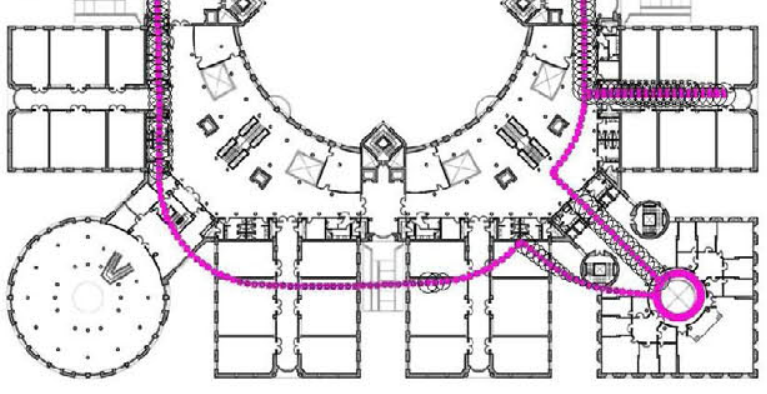

Fig. 16. Trajectory with a long circular path repeated 20 times (at right-bottom floormap) in order to test the magnetically-aided dominant direction correction of MiHDE. a) Results for IEZ, b) HDE estimation, c) iHDE estimation, and d) MiHDE estimation. 